\title{
FACTORES ASOCIADOS A LAS PREFERENCIAS ALIMENTARIAS DE LOS NIÑOS*
}

\section{FACTORS ASSOCIATED WITH CHILDREN'S FOOD PREFERENCES}

\author{
Sugey Elena Anaya-García** \\ Mónica María Álvarez-Gallego***
}

\section{Resumen}

Objetivo. Presentar una revisión bibliográfica de la influencia de las familias y la escuela sobre las preferencias alimentarias de los niños. Su objetivo es comprender aquellos factores asociados a las preferencias alimentarias infantiles. Metodología. Se hizo análisis de información de investigaciones realizadas en el tema con el fin de indagar cómo estamos ante la prevención de la malnutrición en los niños y el fomento de hábitos alimenticios saludables. Resultados. La familia es determinante en la formación de las preferencias alimentarias de los niños, pero también lo es en la aparición de trastornos de la conducta alimentaria. El entorno educativo es fundamental en la promoción de hábitos alimentarios saludables. Conclusiones. En la consolidación de hábitos alimentarios durante la niñez, además de considerar la influencia de la familia y la escuela, deben tenerse en cuenta asuntos como la inapetencia, la neofobia alimenticia y la construcción de la personalidad.

Palabras clave: conducta alimentaria, familia, niños, preferencias alimentarias.

\begin{abstract}
Objective. The main objective is to introduce a literature review of the influence of families and schools on children's food preferences and to understand those factors associated with children's food preferences. Methodology. An analysis of information of the existing research around this topic was carried out in order to investigate condition of of malnutrition prevention in kids and in the promotion of healthy eating habits. Results. The family is a determining factor in the formation of children's dietary preferences, but also in the appearance of eating disorders. The educational environment is fundamental in the promotion of healthy eating habits. Conclusions. In the consolidation of eating habits during childhood, in addition to considering the influence of the family and the school, issues such as lack of appetite, alimentary neophobia and the development of the personality should be taken into account.
\end{abstract}

Key words: children, eating behavior, family, food preferences.

\footnotetext{
* El presente artículo de revisión hace parte del proyecto de investigación "Acompañamiento familiar en la formación de las preferencias alimentarias de los niños”, articulado al macroproyecto "Estructura y Dinámica Interna Familiar con relación a la participación ciudadana”. Grupo de investigación: Educación, Infancia y Lenguas Extranjeras, Universidad Católica Luis Amigó.

** Universidad Católica Luis Amigó. Medellín, Colombia. E-mail: sugey.anayaga@amigo.edu.co -

(1) orcid.org/0000-0002-4166-6277. Google Scholar

**** Universidad Católica Luis Amigó. Medellín, Colombia. E-mail: monica.alvarezga@amigo.edu.co -

(D) orcid.org/0000-0002-3894-0554.
} 


\section{Introducción}

En los últimos años la preocupación ante el aumento de niños con sobrepeso y obesidad se ha tratado de distintas formas en cada país. En el año 2014, 170 países adscritos a la OMS asumieron unos compromisos específicos sobre política e inversión para garantizarles a las personas el acceso a dietas nutricionales saludables. Surge entonces un interrogante: ¿Qué tan efectivos han resultado dichos compromisos? En Colombia anteriormente la mirada se enfocaba solo en personas que presentaban algún tipo de desnutrición, pero la realidad actual nos muestra que factores como: los inadecuados hábitos alimentarios por parte de los padres, el avance de las nuevas tecnologías, los problemas sociales, el aumento de trastornos alimentarios en la población y las nuevas formas de vida, nos obligan a mirar al punto en el que se gestan las preferencias alimentarias: la familia. De acuerdo al Plan Nacional de Seguridad Alimentaria y Nutricional -PNSAN- (2012-2019), Colombia viene desarrollando programas asistenciales de alimentación y nutrición de manera desarticulada, lo que ha dificultado la protección de la población en temas como la malnutrición.

Sobre este tema, se han realizado estudios desde disciplinas como la nutrición, la psicología y la sociología, ya que la alimentación responde a necesidades biológicas, pero también de tipo psicosocial (Villagómez, 2016). De acuerdo a lo anterior, este artículo tiene como finalidad revisar investigaciones a nivel internacional, nacional y local que guarden relación con el tema planteado y permitan vislumbrar aquellos factores no solo familiares sino también de otra índole que pudieran estar afectando la formación de preferencias alimentarias en los niños.

Las investigaciones al respecto han coincidido en que la intervención que se haga en educación nutricional durante los primeros años de vida influirá significativamente en las preferencias nutricionales y los estilos de vida saludables de los niños. Estudios como los de Cárcamo y Mena (2006), López et al. (2007), García, Liévano, Liévano, Leclercq y Moreno (2008), Cabello y Reyes (2011), González et al. (2012), Franco (2013), Silva, Jiménez y Hernández (2013), Arboleda (2014) y Campos y Reyes (2014), aseguran que dentro de la familia la madre es la responsable, en la mayoría de los casos, de transmitir a sus hijos las prácticas nutricionales y de esta manera incidir en sus comportamientos alimentarios. Teniendo en cuenta que la mayoría de estos estudios se llevaron a cabo en México y que el tipo de metodología utilizada fue tanto cuantitativa como cualitativa, utilizando en su mayoría cuestionarios y entrevistas respectivamente, se encontró que un aspecto poco estudiado en estas investigaciones es el tipo de educación nutricional con que cuentan estas madres, así como el origen de sus creencias alimentarias dada la influencia que ejercen dentro de las familias.

En suma, además de la madre, los estudios señalan que el estado psicológico del niño, las relaciones sociales entre sus pares, los profesores, el Gobierno, etc., influyen en la formación de las preferencias alimentarias de los niños y se podría decir que son los responsables de 
Factores asociados a las preferencias alimentarias de los niños

promover prácticas alimenticias sanas que coadyuvan a mitigar el aumento en la prevalencia de la malnutrición en el mundo.

Los resultados encontrados invitan a educar a las familias y profesores a partir de información verídica y se concluye, a partir del presente artículo, que se hace necesario involucrar en las investigaciones a otros actores como los cuidadores o responsables de la alimentación, así como el círculo social del niño, además de abordar la manera en que se imparten las normas cuando el niño se encuentra solo y/o acompañado por sus padres, pues es poca la evidencia encontrada al respecto. Para el caso de Colombia y a pesar de que los estudios encontrados se centran en identificar la frecuencia de consumo de alimentos y comprender las estrategias que utilizan las familias para satisfacer esa necesidad humana, como lo es el alimentarse, hacen falta investigaciones que den muestra de la aplicación de estrategias para el fomento de hábitos alimentarios saludables y su efectividad.

El proyecto de investigación "Acompañamiento familiar en la formación de las preferencias alimentarias de los niños" se desarrolla a partir del enfoque cualitativo de corte de tipo descriptivo.

Ahora bien, para la construcción de este artículo se siguieron tres pasos que permitieron establecer unos criterios para buscar la información, organizarla, analizarla y luego reunir los resultados más importantes. El primer paso se basó en establecer los criterios de búsqueda, los cuales fueron: a) artículos derivados de proyectos de investigación, b) artículos cuya publicación estuviera comprendida entre 2010-2017, c) se tuvieron en cuenta las categorías establecidas para el rastreo de los artículos, las cuales son: preferencias alimentarias, familia y niños.

En el segundo paso, y después de establecer los criterios de selección, se procedió a realizar el rastreo. La búsqueda se realizó en revistas halladas en bases de datos como: SciELO, EBSCO, ScienceDirect, Redalyc y Google Académico. Se obtuvieron 70 publicaciones de las cuales se rechazaron 20, ya que se centraban en adolescentes o su enfoque principal era la valoración nutricional de los niños, lo que incumplía con los criterios de búsqueda establecidos. Para los resultados y discusión del presente artículo se seleccionaron 50 estudios que cumplían con la finalidad del mismo.

Finalmente, para la redacción de este artículo se organizó la información por medio de una matriz de análisis bibliográfico con los siguientes ítems: título del artículo (autor, año), tema, objetivo, palabras clave, método de análisis y hallazgos del estudio. Seguidamente se agruparon los estudios en tres núcleos temáticos: la familia en las preferencias alimentarias, papel de la escuela y, por último, todos aquellos factores que inciden en las preferencias alimentarias. 


\section{Discusión}

\section{Influencia familiar en las preferencias alimentarias infantiles}

Las primeras posturas de los autores revisados, en cuanto a las preferencias alimentarias en los niños, señalan a "la familia como un grupo primario en el aprendizaje de hábitos, costumbres, normas y valores que ubican la alimentación como un mediador de las prácticas de socialización” (Garzón y Barreto, 2013, p. 91). Los autores mencionados anteriormente, escogieron para su estudio de tipo descriptivo 40 familias de un sector urbano en la ciudad de Bogotá, aplicaron una encuesta con preguntas abiertas y cerradas, y entre sus principales resultados obtuvieron que la alimentación es para compartir en familia, que la madre es el eje central de ello y que los alimentos ligth los definen como nutriente.

Black y Creed-Kanashiro (2012) en su investigación examinaron las conductas de alimentación temprana, encontrando que los primeros dos años de vida de un niño son un tiempo especial para ayudar a las familias a establecer hábitos alimentarios saludables y de esta manera evitar la aparición de algún tipo de malnutrición, mientras que De La Cruz (2015) establece la necesidad de abordar el tema integralmente desde aspectos como la alimentación y nutrición en educación, el valor de la dieta para la niñez, los hábitos alimentarios, entre otros. Cabe resaltar la propuesta de este último autor de involucrar disciplinas como la antropología, historia, ambiente, psicología y economía, en estas temáticas.

En concordancia, se invita a los padres a tener una participación activa en sus familias, que les permita involucrarse en la vida de sus hijos brindándoles amor y afecto, llamado que realizan Carrillo, Bermúdez, Suárez, Gutiérrez y Delgado (2016), quienes sugieren además que:

\footnotetext{
(...) una mayor comprensión del rol de los papás y de su involucramiento en la familia y con los hijos contribuirá al desarrollo de programas y las políticas orientadas hacia todos los miembros de la familia y hacia los diferentes sistemas de relación en el contexto familiar. (p. 102)
}

En cuanto a la formación de hábitos alimenticios saludables, Rodrigo, Soriano y Aldas (2016) pudieron comprobar que la adherencia de la dieta mediterránea en padres y niños aumenta significativamente los conocimientos en hábitos saludables, así como el consumo de fruta, verdura y pescado. Lo anterior determina que la intervención en educación alimentaria mejora los conocimientos de los padres, quienes son los responsables de la alimentación de sus hijos.

Pero, ¿qué pasa si la información nutricional que encuentran los padres es quizás contradictoria? Castrillón y Giraldo (2014) sugieren que el mejoramiento de las prácticas alimentarias debe incluir elementos psicológicos y psicofisiológicos de la alimentación, dado que los problemas 
de este tipo que se presentan en la infancia "lleva[n] a estados emocionales bajo los cuales se toman decisiones que afectan aún más las conductas alimentarias, lo que puede llevar a mayores dificultades en la interacción” (p. 69). Una de las fuentes a la cual acuden los padres en busca de información es al pediatra. Solans (2012) enuncia que "desde la práctica médica, se pueden trabajar las brechas entre el conocimiento normativo y las prácticas alimentarias ofreciendo explicaciones sobre la calidad nutricional de algunos productos alimentarios y los significados asociados a su consumo” (p. 142).

En investigaciones sobre problemas de tipo nutricional como los trastornos de la conducta alimentaria (TCA), Rodríguez, Hernández, Bolaños, Ruiz y Jáuregui (2015a) advierten que existe una influencia del entorno familiar en el desarrollo de los TCA, ya que aspectos como el peso y la figura se transmiten de padres a hijos. Ahora, Ruíz, Vásquez, Mancilla, Viladrich y Halley (2013) concluyen que las personas con TCA están presentes en ambientes familiares desfavorecidos. Sin embargo, otros hallazgos como los de Martin, Dovey y Arcelus (2012) mencionan que a pesar de que los trastornos de la ingestión alimentaria de la infancia (TIAI) se encuentran separados de los TCA, no existe suficiente información para abordarlos y podrían ser investigados con mayor profundidad, puesto que las condiciones de las relaciones interpersonales a la hora de las comidas actuarían como factores contribuyentes o de mantenimiento de los TIAI. Solo uno de los estudios antes mencionados (Rodríguez et al., 2015a) incluye la influencia de las redes sociales en este tipo de trastornos, por lo que podría convenir un mayor interés en investigaciones de esta índole, dada la fuerte utilización de estos medios en la actualidad. En Colombia es escasa la producción de estudios con esta naturaleza que podrían contribuir a un mejor abordaje de estos problemas alimentarios a nivel nacional.

Finalmente, la población en estas investigaciones en definitiva la conformaron las familias, pero con cada uno de sus miembros (padres, madres e hijos) de una manera aislada, aplicando metodologías que permitían análisis tanto descriptivos como estadísticos, presentándose un vacío en estudios que incluyan a la familia como un solo conjunto, constitutivo de eje principal de la sociedad.

\section{Incidencia del entorno educativo en las preferencias alimentarias de los niños}

Dentro de los aportes encontrados acerca del papel de la escuela y los profesores en las conductas alimentarias del escolar, Vio, Salinas, Montenegro, González y Lera (2014) señalan que "una intervención educativa participativa, con uso de TIC y de corta duración en profesores y alumnos de pre básica y básica, puede producir cambios positivos en el estado nutricional, mejorando significativamente el conocimiento y consumo de alimentos saludables" (p. 1298). 
Otros estudios resaltan la necesidad de educar a los niños y sus familias en educación nutricional desde la escuela y en promoción de estilos de vida saludables, tales como: García, Pardío, Arroyo y Fernández (2008), Vio, Salinas, Lera, González y Huenchupán (2012), Alvear et al. (2013), Lera, Salinas, Fretes y Vio (2013), Orozco y Ruiz (2014), Sánchez, Reyes y González (2014) y Vio et al. (2015). Se resalta el gran valor que debe tener el entorno educativo en la intención de reducir las cifras de malnutrición en los niños.

En este punto, es importante considerar el estudio de Fretes, Salinas y Vio (2013) en donde, al llevar a cabo una intervención educativa en alimentación y nutrición a las familias de niños preescolares y escolares, se pudo evidenciar que "es posible realizar cambios en los hábitos alimentarios de las familias, con la implementación de una intervención educativa que incluya talleres de cocina y materiales audiovisuales" (p. 37). Por otro lado, guardando relación con lo anterior, Abril et al. (2012) enuncian que este tipo de intervenciones deben ser continuas para poder incidir integralmente en el desarrollo cognitivo del niño e involucrar a los padres y maestros a fin de garantizar su sustentabilidad.

Por su parte, Del Campo, Vara y Navarro (2010) analizaron los hábitos alimenticios en alumnos y la tarea pedagógica de los docentes, después de intervenirlos por medio de una investigación de acción participativa en educación alimentaria y nutricional, como resultado se obtuvo un impacto positivo, ya que los docentes incluyeron en su proyecto educativo institucional un trabajo sobre alimentación y nutrición, que involucró al quiosco escolar y a los padres de familia. En igual medida, Maldonado (2013) determinó que un programa de educación nutricional contribuye a mejorar los conocimientos, actitudes y prácticas relacionadas con el consumo de frutas y verduras en los niños.

Es pertinente entonces mencionar que uno de los problemas que se pueden encontrar en las instituciones educativas es la falta de conocimiento por parte de las familias y los profesores en temas de alimentación y nutrición, tal como lo señala Restrepo (2007), lo cual no favorece el fomento y práctica de hábitos saludables. En consecuencia, podría suponerse que el porcentaje de niños con sobrepeso tiende a aumentar, tal como lo encontraron Castillo et al. (2012) en su estudio, cuyos resultados mostraron un desequilibrio alimenticio en la población infantil por exceso de proteínas, carbohidratos, colesterol y déficit en la ingesta de micronutrientes.

Los estudios anteriormente mencionados muestran claramente una preocupación hacia las intervenciones que reciben o no los estudiantes, sus familias y profesores en hábitos alimentarios saludables, situación que pudiera ser aprovechada para mitigar el incremento de sobrepeso y obesidad en la población a nivel mundial. 
Factores asociados a las preferencias alimentarias de los niños

\section{Otros factores asociados a las preferencias alimentarias}

Es necesario comprender la complejidad de los múltiples factores que rodean la alimentación y cómo estos influyen en las preferencias alimentarias de los niños. De todo esto se desprende que:
(...) son múltiples los factores que estimulan las sensaciones de hambre
y saciedad y condicionan la adopción de los hábitos alimentarios en los niños. Desde aspectos individuales como los fisiológicos y los genéticos y los externos como los del entorno y la familia. (Ducuara, 2011, p. 163)

En complemento con lo anterior, Díaz (2014) enuncia en su artículo de revisión, y en donde utilizó estudios que investigaran aspectos relacionados con el comportamiento alimentario infantil, que existen otros factores que deben ser tenidos en cuenta a la hora de realizar un análisis integral a la problemática nutricional actual tales como: las estrategias de consumo de alimentos por parte de los padres, la influencia de los pares, las emociones y experiencias relacionadas con alimentos particulares.

Al respecto, Macías, Gordillo y Camacho (2012) consideran que si bien la familia ejerce una fuerte influencia en los patrones de consumo de los niños, se deben tener en cuenta los factores de tipo fisiológico y social, corroborando el momento de la alimentación como un proceso integral que se debe aprovechar al máximo al irlos formando como sujetos socializadores.

Ahora bien, los ingresos económicos de las familias entran a determinar el acceso, la variedad y frecuencia de los alimentos (Restrepo y Maya, 2005), y no solo esto, también se encuentran como "determinantes sociales de la alimentación en los estratos, el nivel educativo, los gustos, la cultura y la influencia del medio social” (Belalcázar y Tobar, 2013, p. 40).

Otros autores como Miqueleiz et al. (2014) en su estudio se propusieron investigar la posible asociación de patrones alimentarios relacionados con la obesidad y la posición socioeconómica, resaltando que:

(...) tanto en la infancia como en la adolescencia la magnitud de la razón de prevalencia muestra un gradiente socioeconómico inverso en todos los consumos de alimentos investigados: la menor y la mayor razón de prevalencia se observa en los sujetos de familias de posición socioeconómica más alta y más baja, respectivamente. (p. 433) 
Por su parte, Maya y Naranjo (2015) alertan sobre el interés que se le debe dar al temperamento de cada niño y su estado emocional con relación a las prácticas alimentarias, pues mencionan que "la ansiedad, la tristeza, los enfados provocan generalmente una ausencia de apetito. Mientras que situaciones de alegría, satisfacción, optimismo, etc., producen un efecto positivo en la alimentación” (p. 41).

En esa misma dirección, Maiz, Maganto y Balluerka (2014) establecieron en su revisión acerca de neofobia alimentaria, cuyo significado es miedo a probar nuevos alimentos, que esta "tiene un gran impacto y una repercusión directa en el consumo de diferentes alimentos y, especialmente, en la ingesta de frutas y verduras, confirmando que los niños neofóbicos presentan dietas menos saludables que sus compañeros neofílicos” (p. 150).

Autores como Rodríguez et al. (2015b) coinciden en que la neofobia se asocia a una disminución en el consumo de verduras y frutas de los escolares.

Por otra parte, existen algunos estudios sobre políticas alimentarias que develan diferentes posturas. Acosta (2014) observó en los niños que reciben intervención alimentaria en el hogar un consumo mayor de energía proveniente de los cereales, concluyendo que "estas acciones asistenciales deben ir acompañadas de la complementación de intervenciones que promuevan de manera universal, continua y permanente el acceso a una alimentación adecuada” (p. 330).

Asimismo, Ibáñez y Juergo (2012) corroboran lo dicho anteriormente dado que ponen de manifiesto la manera en que las políticas alimentarias son protagonistas de la desigualdad social, además de cómo la asistencia brindada ante los problemas alimentarios desvía lo social e institucional de su nodo estructural constitutivo.

En cuanto a la seguridad alimentaria, Taborda, Pérez y Berbesi (2011) encontraron que esta se asocia con la funcionalidad familiar y la desnutrición crónica, elementos que se convierten en factores de riesgo. De manera semejante, Vega, Shamah, Peinador, Méndez y Melgar (2014) encontraron una relación entre una menor variedad de alimentos y mayor inseguridad alimentaria, lo que posiblemente denota un reemplazo de la proteína en hogares con inseguridad alimentaria.

Finalmente, Salinas y Vio (2011) junto con Hernández et al. (2015) coinciden en que es necesaria la presencia de otros sectores y gremios que trabajen conjuntamente en el fomento de hábitos alimentarios saludables entre los niños, tales como el sector salud, educación, la academia y el sector privado, que permitan poner en marcha políticas de promoción de la salud. 


\section{Conclusiones}

Teniendo en cuenta las investigaciones revisadas, se pone en evidencia que dentro de las familias se ejerce una fuerte influencia sobre las preferencias alimentarias de los niños. Según Domínguez, Olivares y Santos (2008), el patrón alimentario familiar es relevante en el comportamiento alimentario del niño, convirtiéndose en un aspecto importante en la prevención de la obesidad infantil.

Pero, además de la prevención de la obesidad infantil debe prestarse atención a la malnutrición en la infancia. Para intervenir esta última, la familia se constituye en una fuente de conocimientos que bien podrían llegar a ser positivos o negativos, según la educación con que cuenta en hábitos alimentarios.

En diversos estudios fue recurrente la aparición de la madre como la persona que toma las decisiones más importantes en cuanto al proceso alimentario en su familia, en su mayoría es quien determina qué se come en el hogar y bajo qué circunstancias, reafirmando que aún persiste esa labor de preparación y servida de los alimentos por parte de ellas.

Así mismo, el nivel educativo de los padres y sus ingresos económicos repercuten en el tipo de alimentos y su accesibilidad, situación que podría influir inevitablemente en el estado nutricional de cada uno de los integrantes de la familia.

Pero, también las escuelas tienen un rol importante en el fomento de hábitos alimenticios saludables. De hecho, se sugiere la implementación de estrategias como la educación nutricional en las mismas, que permitan adquirir en los niños información confiable y verídica acerca de lo que consumen. Este tipo de planteamiento fue reiterativo en varias de las fuentes consultadas. Se propone entonces educación en normas alimentarias sanas desde el inicio de la vida y de la etapa escolar, con el fin de ayudar a evitar la aparición de problemas que afecten el desarrollo personal.

Con relación a los aspectos metodológicos, los estudios son en su mayoría de corte transversal, ya que combinan elementos cualitativos y cuantitativos que permiten la aplicación de instrumentos como entrevistas, talleres, pretest y postest. Pocos estudios incluyeron a padres, alumnos y profesores de manera conjunta, lo que revela una debilidad en cuanto a la construcción de información ya que no permite comprender a fondo el tema de preferencias alimentarias. Es para tener en cuenta el aporte valioso que nos proporcionan los estudios realizados en Chile, en donde abordan de manera interesante el tema de los hábitos y consumo alimentario en el ámbito educativo. 
Es latente la necesidad de implementar políticas alimentarias enfocadas en ayudar a la población y a las familias en los asuntos relacionados con la nutrición. Si bien existen, como es el caso de Chile y su programa nacional de alimentación completaria; de Colombia con su política 'De Cero a Siempre' que aborda de manera integral la atención de niños de la primera infancia; de Brasil con el programa nacional de alimentación escolar, entre otros. A pesar de estos esfuerzos, los programas aún se quedan cortos porque no existe un acompañamiento claro y efectivo que los complemente, por lo que se limitan a ofrecer servicios alimentarios que se desvían de la atención y seguimiento de manera integral. Para el caso de Colombia, los patrones de actividad física y alimentación están siendo afectados por la influencia política y mediática de grandes empresas de alimentos y bebidas ultraprocesados, situación que repercute en la salud infantil (Gómez et al., 2012).

Se observa un llamado claro y urgente para llevar a cabo programas de promoción de alimentación sana, que involucren diferentes actores, incluida la sociedad con el fin de lograr una articulación integral que obtenga mejores resultados.

En cuanto a los factores diferentes a la familia y la escuela que posiblemente inciden en la formación de prácticas alimentarias adecuadas en la niñez, se hace muy evidente el contexto social, pues es ahí donde los niños se hacen vulnerables a información muchas veces equívoca, proveniente de sus pares, de los medios de comunicación y de las personas que los rodean.

Sobre la misma base se identificaron aspectos de tipo intrínseco (género, edad, genética, etc.) y extrínseco (influencia de pares, emociones, entre otros) en los niños, que es factible repercutan en las preferencias alimentarias. Por tanto, diferentes investigaciones sugieren una mayor indagación en cuanto a los patrones alimenticios en la infancia ya que se podría incurrir en errores a la hora de tomar medidas que apunten a una necesidad no priorizada en ellos.

Finalmente, estas investigaciones muestran un interés muy marcado en brindar a las familias educación nutricional que permita la transmisión de hábitos alimentarios adecuados en los niños, ya que de lo contrario tienden a replicarse patrones y costumbres alimenticias que quizás no les permitan a los niños crecer y desarrollarse de una manera óptima. Además, se deben incluir los cuidadores, que en su mayoría son las abuelas y los amigos de los niños, en los estudios que aborden este tema.

Se reconoce que serían de gran ayuda investigaciones que den muestra de todos aquellos actos que se presentan alrededor de la alimentación, como son las normas, las costumbres arraigadas de generación en generación en torno a los alimentos consumidos, la influencia de la tecnología que actualmente penetra en todos los ámbitos, entre muchos otros que podrían estar quedando por fuera a la hora de indagar acerca de las preferencias alimentarias de los niños. 
Factores asociados a las preferencias alimentarias de los niños

Adicionalmente, se propone investigar de qué manera la tecnología puede ser utilizada en el fomento de prácticas alimentarias saludables y cómo los padres están asumiendo la responsabilidad de la alimentación de su hijo, si se vislumbra poca presencia de ellos en este espacio.

\section{Referencias}

Abril, E., Rascón, C., Bonilla, P., Hernández, H., Cuevas, S. y Arenas, L. (2012). Promoción de hábitos saludables en escolares de Hermosillo, Sonora, México. Revista Cubana de Higiene y Epidemiología, 50 (3), 354-364. Recuperado de http://scielo.sld.cu/pdf/hie/ v50n3/hie10312.pdf

Acosta, L. (2014). Características de la dieta en niños de 2 a 5 años según la recepción de programas alimentarios en el hogar. Argentina. Rev. Gerenc. Polit. Salud, 13 (27), 319-333. Recuperado de http://dx.doi.org/10.11144/Javeriana.rgyps13-27.cdna

Alvear, M., Yamamoto, L., Morán, C., Solís, M., Torres, P., Juárez, M... Ferreira, A. (2013). Consumo alimentario dentro y fuera de la escuela. Revista Médica del Instituto Mexicano del Seguro Social, 51 (4), 450-455. Recuperado de http://www.uacm.kirj.redalyc.redalyc. org $/$ articulo.oa?id $=457745490018$

Arboleda, L. (2014). Dinámicas y estrategias alimentarias instauradas en hogares de Medellín. Rev. Fac. Nac. Salud Pública, 32 (3), 282-289. Recuperado de http: / / aprendeenlinea.udea. edu.co/revistas/index.php/fnsp/article/view/17849

Belalcázar, D. y Tobar, L. (2013). Determinantes sociales de la alimentación en familias de estratos 4, 5 y 6 de la localidad de Chapinero de Bogotá D.C. Rev. Fac. Nac. Salud Pública, 31 (1), 40-47. Recuperado de http: / / aprendeenlinea.udea.edu.co/revistas/index.php/ fnsp/article/view/12592/13306

Black, M. y Creed-Kanashiro, H. (2012). ¿Cómo alimentar a los niños? La práctica de conductas alimentarias saludables desde la infancia. Rev Perú Med Exp Salud Pública, 29 (3), 373-378. Recuperado de http://www.scielo.org.pe/scielo.php?script=sci_arttext\&pid $=$ S1726-46342012000300013

Cabello, M. y Reyes, D. (2011). Percepción de las madres de niños con obesidad sobre los hábitos alimenticios y sus responsabilidades en la alimentación de los hijos. Revista salud pública y nutrición, 12 (1). Recuperado de http://www.medigraphic.com/pdfs/ revsalpubnut/spn-2011/spn111c.pdf

Campos, N. y Reyes, I. (2014). Preferencias alimentarias y su asociación con alimentos saludables y no saludables en niños preescolares. Acta de Investigación psicológica, 4 (1), 1385-1397. Recuperado de http://www.sciencedirect.com/science/article/pii/ S2007471914703825 
Cárcamo, G. y Mena, C. (2006). Alimentación saludable. Horizontes educacionales, 11 (1). Recuperado de http: / / www.redalyc.org/articulo.oa?id=97917575010

Carrillo, S., Bermúdez, M., Suárez, L., Gutiérrez, M. y Delgado, X. (2016). Percepciones del padre sobre su rol e involucramiento en la familia: un estudio cualitativo en una muestra colombiana. Revista costarricense de psicología, 35 (2), 101-118. Recuperado de http://www.scielo.sa.cr/scielo.php?script=sci_arttext\&pid=S165929132016000200037\&lng $=\mathrm{en} \& \operatorname{tn} g=\mathrm{en}$

Castillo, O., Velázquez, G., Uresti, R., Mier, N., Vásquez, M. y Ramírez, J. (2012). Estudio de los hábitos alimentarios de niños de 4-6 años de Reynosa, Tamaulipas (México). CyTA - Journal of Food, 10 (1), 5-11. Recuperado de http: / / dx.doi.org/10.1080/194763 37.2010 .524941

Castrillón, I. y Giraldo, O. (2014). Prácticas de alimentación de los padres y conductas alimentarias en niños: ¿Existe información suficiente para el abordaje de los problemas de alimentación? Revista de psicología Universidad de Antioquia, 6 (1), 57-74. Recuperado de http://aprendeenlinea.udea.edu.co/revistas/index.php/psicologia/article/view/21617

De La Cruz, E. E. (2015). La educación alimentaria y nutricional en el contexto de la educación inicial. Paradigma, 36 (1), 161-183. Recuperado de http://revistas.upel.edu.ve/index. $\mathrm{php} /$ paradigma/article/view/2663

Del Campo, M., Vara, M. y Navarro, A. (2010). Educación alimentaria-nutricional (EAN) en la enseñanza primaria municipal de Córdoba. Una experiencia en investigación-acción participativa (IAP). Diaeta (B. Aires), 28 (132), 15-22. Recuperado de http: / / www.scielo. org.ar/pdf/diaeta/v28n132/v28n132a03.pdf

Díaz, M. (2014). Factores influyentes en el comportamiento alimentario infantil. Rev. Fac. Med., 62 (2), 237-245. Recuperado de http://www.scielo.org.co/pdf/rfmun/v62n2/ v62n2a10.pdf

Domínguez, P., Olivares, S. y Santos, J. L. (2008). Influencia familiar sobre la conducta alimentaria y su relación con la obesidad infantil. Archivos latinoamericanos de nutrición, 58 (3), 249-255. Recuperado de http: / / repositorio.uchile.cl/bitstream/handle/2250/123925 / Dominguez_p.pdf?sequence $=1$

Ducuara, P. (2011). Consideraciones sobre la inapetencia infantil y la adopción de hábitos alimentarios saludables. Revista Ces medicina, 25 (2), 153-168. Recuperado de http: / /www.scielo.org.co/pdf/cesm/v25n2/v25n2a04.pdf

Franco, S. (2013). Cambios y permanencias en el proceso de alimentación familiar. Revista Latinoamericana de Estudios de Familia, 5, 83-105. Recuperado de http:/ / web.b.ebscohost. com / ehost/pdfviewer/pdfviewer?vid=3\&sid=00f42e08-bfad-4b84-be 38 ec69eab06a8f\%40sessionmgr101\&hid=124 
Fretes, G., Salinas, J. y Vio, F. (2013). Efecto de una intervención educativa sobre el consumo de frutas, verduras y pescado en familias de niños preescolares y escolares. Archivos latinoamericanos de nutrición, 63 (1), 37-45. Recuperado de http://renhyd.org/index. $\mathrm{php} / \mathrm{renhyd} /$ article/view/182

García, G., Liévano, G., Liévano, M., Leclercq, M. y Moreno, D. (2008). Caracterización de hábitos alimentarios y estilos de vida de los niños del Jardín Vaticanitos, Bogotá, D.C. Perspectivas en nutrición humana, 10 (2), 143-152. Recuperado de http: / www.scielo.org. $\mathrm{co} / \mathrm{pdf} / \mathrm{penh} / \mathrm{v} 10 \mathrm{n} 2 / \mathrm{v} 10 \mathrm{n} 2 \mathrm{a} 3 . \mathrm{pdf}$

García, M., Pardío, J., Arroyo, P. y Fernández, V. (2008). Dinámica familiar y su relación con hábitos alimentarios. Estudios sobre las culturas contemporáneas, 14 (27), 9-46. Recuperado de http: / / www.scielo.cl/scielo.php?pid=S0717-75182011000200001\&script $=$ sci_arttext

Garzón, C. y Barreto, I. (2013). Prácticas alimentarias y significados de alimentos light de familias de un sector urbano de Bogotá. Revista Suma Psicológica, 20 (1), 89-99. Recuperado de http: / / publicaciones.konradlorenz.edu.co/index.php/sumapsi/article/ view/1062/808

Gómez, L., Ibarra, M., Lucumí, D., Arango, C., Parra, A., Cadena, Y... Parra, D. (2012). Alimentación no saludable, inactividad física y obesidad en la población infantil colombiana: un llamado urgente al Estado y la sociedad civil para emprender acciones efectivas. Global Health Promotion, 19 (3), 87-92. Recuperado de https://www.researchgate.net/ publication/262108142_Alimentacion_no_saludable_inactividad_fisica_y_obesidad_ en_la_poblacion_infantil_colombiana_Un_llamado_urgente_al_estado_y_la_sociedad_ civil_para_emprender_acciones_efectivas

González, E., Aguilar, M., García, C., García, P., Álvarez, J., Padilla, C. y Ocete, E. (2012). Influencia del entorno familiar en el desarrollo del sobrepeso y la obesidad en una población de escolares de Granada (España). Nutrición Hospitalaria, 27 (1), 177-184. Recuperado de http://scielo.isciii.es/scielo.php?script=sci_arttext\&pid=S0212$16112012000100021 \& \operatorname{lng}=$ es\&tlng $=$ es.

Hernández, E., Severiche, D., Romero, D., López, M., Espitia, V. y Rodríguez, A. (2015). Promoción de alimentación saludable en hogares comunitarios infantiles del municipio de Sopó (Cundinamarca, Colombia) bajo la estrategia de Atención Primaria en Salud. Salud Uninorte, 31 (3), 514-524. Recuperado de http://rcientificas.uninorte.edu.co/index. $\mathrm{php} / \mathrm{salud} / \mathrm{article} / \mathrm{view} / 7632 / 8310$

Ibáñez, I. y Juergo, J. (2012). Encima que les dan, eligen, políticas alimentarias, cuerpos y emociones de niños/as de sectores populares. Revista latinoamericana de estudios sobre cuerpos, emociones y sociedad, 8, 29-42. Recuperado de http: / /www.relaces.com.ar/index. $\mathrm{php/relaces/article/viewArticle/156}$ 
Lera, L., Salinas, J., Fretes, G. y Vio, F. (2013). Validación de un instrumento para evaluar prácticas alimentarias en familias chilenas de escolares de 4 a 7 años. Nutrición Hospitalaria, 28 (6), 1961-1970. Recuperado de http://scielo.isciii.es/pdf/nh/ v28n6/27originalpediatria05.pdf

López, J., Vázquez, V., Bolado, V., González, J., Castañeda, J., Robles, L... Comuzzie, A. (2007). Influencia de los padres sobre las preferencias alimentarias en niños de dos escuelas primarias con diferente estrato económico. Gac Méd Méx, 143 (6), 463-469. Recuperado de http: / /www.medigraphic.com/pdfs/gaceta/gm-2007/gm076b.pdf

Macías, A., Gordillo, L. y Camacho, E. (2012). Hábitos alimentarios de niños en edad escolar y el papel de la educación para la salud. Rev Chil Nutr, 39 (3), 40-43. Recuperado de http://aprendeenlinea.udea.edu.co/revistas/index.php/nutricion/article/ view/9340/8597

Maiz, E., Maganto, C. y Balluerka, N. (2014). Neofobia y otros trastornos restrictivos alimentarios en la infancia y consumo de frutas y verduras: revisión: Neophobia and Other. Revista Española de Nutrición Comunitaria, 20 (4), 150-157. Recuperado de https: / /www.researchgate.net/publication/277984953

Maldonado, L. (2013). Evaluación del cambio en conocimientos, actitudes y prácticas (CAPs) sobre el consumo de verduras y frutas en niños de edad preescolar de una escuela pública de la ciudad capital de Guatemala. Revista de la Universidad del Valle de Guatemala, 25, 29-38. Recuperado de http://www.scielo.cl/scielo.php?pid=S0717$75182011000200001 \&$ script $=$ sci_arttext

Martin, C., Dovey, T. y Arcelus, J. (2012). Trastornos del la ingestión alimentaria de la infancia o la niñez: Una revisión. Revista Mexicana de Trastornos Alimentarios, 3, 8998. Recuperado de http://www.scielo.org.mx/scielo.php?script=sci_arttext\&pid $=$ S2007-15232012000200002

Maya, E. y Naranjo, J. (2015). Estudio de casos sobre conducta y hábitos alimentarios en niños de Educación Infantil. Reidocrea, monográfico, 32-42. Recuperado de http: / / hdl.handle.net/10481/37113

Miqueleiz, E., Lostao, L., Ortega, P., Santos, J., Astasio, P. y Regidor, E. (2014). Patrón socioeconómico en la alimentación no saludable en niños y adolescentes en España. Atención primaria, 46 (8), 433-439. Recuperado de http://www.sciencedirect.com/ science/article/pii/S0212656714000304

Orozco, J. M. y Ruiz, A. C. (2014). Efectividad de un programa educativo para mejorar conocimientos sobre alimentación saludable en los niños y niñas de 5 años de una I.E.I. de Chiclayo. Rev. Salud \&Vida Sipanense, 1 (1), 16-29. Recuperado de http: / / revistas.uss.edu. pe/index.php/SVS/article/view/68 
Factores asociados a las preferencias alimentarias de los niños

Restrepo, S. (2007). Percepciones frente a la alimentación y nutrición del escolar. Perspectivas en nutrición humana, 9 (1), 23-35. Recuperado de http://aprendeenlinea.udea.edu.co/ revistas/index.php/nutricion/article/view/9340

Restrepo, S. y Maya, M. (2005). La familia y su papel en la formación de los hábitos alimentarios en el escolar. Un acercamiento a la cotidianidad. Boletín de antropología, 19 (36), 127 148. Recuperado de https://aprendeenlinea.udea.edu.co/revistas/index.php/boletin/ article/view/6919/6333

Rodrigo, S., Soriano, J. y Aldas, J. (2016). Valoración de la efectividad de la educación alimentaria en niños preescolares, padres y educadores. Rev Esp de Nutr Hum Diet, 20 (1), 32-39. Recuperado de http: / / scielo.isciii.es/pdf/renhyd/v20n1/original4.pdf

Rodríguez, M., Hernández, J., Bolaños, P., Ruiz, I. y Jáuregui, I. (2015a). Alimentación familiar y percepción de riesgo en trastornos de la conducta alimentaria. Nutrición Hospitalaria, 32 (4), 1786-1795. Recuperado de http://www.aulamedica.es/gdcr/index.php/nh/ article/view/9635/pdf_8433

Rodríguez, A., Patiño, B., Urquidez, R., Vidaña, M., Periago, M., Ros, G. y Martínez, E. (2015b). Neofobia alimentaria: impacto sobre los hábitos alimentarios y aceptación de alimentos saludables en usuarios de comedores escolares. Nutrición Hospitalaria, 31 (1), 260-268. Recuperado de http: / / www.redalyc.org/articulo. oa?id=309232878026

Ruíz, A., Vásquez, R., Mancilla, J., Viladrich, C. y Halley, M. (2013). Factores familiares asociados a los Trastornos Alimentarios: una revisión. Revista mexicana de trastornos alimentarios, 4, 45-57. Recuperado de http:/ /www.sciencedirect.com/science/article/ $\mathrm{pii} / \mathrm{S} 200715231371992 \mathrm{X}$

Salinas, J. y Vio, F. (2011). Programas de salud y nutrición sin política de Estado: el caso de la promoción de salud escolar en Chile. Rev Chil Nutr, 38 (2), 100-116. Recuperado de http: / / www.scielo.cl/scielo.php?pid=S0717-75182011000200001\&script $=$ sci_arttext

Sánchez, R., Reyes, H. y González, M. (2014). Preferencias alimentarias y estado de nutrición en niños escolares de la Ciudad de México. Bol Med Hosp Infant Mex, 71 (6), 358-366. Recuperado de http://www.sciencedirect.com/science/article/pii/ S1665114615000088

Silva, C., Jiménez, B. y Hernández, A. (2013). Factores relacionados con las prácticas maternas de control alimentario en preescolares. Acta de Investigación Psicológica, 3 (3), 1298-1310. Recuperado de http://www.sciencedirect.com/science/article/pii/ S2007471913709682 
Solans, A. (2012). Promoción de hábitos saludables en la alimentación de los niños. ¿Qué le pasa al pediatra? Arch Argent Pediatr, 110 (2), 137-143. Recuperado de http: / / www. scielo. org.ar/scielo.php?script $=$ sci_arttext\&pid $=$ S0325-00752012000200010

Taborda, P., Pérez, M. y Berbesi, D. (2011). Funcionalidad familiar, seguridad alimentaria y estado nutricional de niños del Programa Departamental de Complementación Alimentaria de Antioquia. Revista Ces medicina, 25 (1), 6-19. Recuperado de http: / / www.redalyc.org/articulo.oa?id=261119568002

Vega, M., Shamah, T., Peinador, R., Méndez, I. y Melgar, H. (2014). Inseguridad alimentaria y variedad de la alimentación en hogares mexicanos con niños menores de cinco años. Salud pública de México, 56 (1), 21-30. Recuperado de http://www.scielo.org.mx/pdf/spm/ v56s1/v56s1a5.pdf

Villagómez, P. (2016). Entre lo que se debe y lo que se puede: percepción y satisfacción de necesidades alimentarias en la ciudad de México. Acta sociológica, 70, 99-128. Recuperado de http:/ /www.sciencedirect.com/science/article/pii/S0186602817300051

Vio, F., Salinas, J., Lera, L., González, C. y Huenchupán, C. (2012). Conocimientos y consumo alimentario en escolares, sus padres y profesores: un análisis comparativo. Rev Chil Nutr, 39 (3), 34-39. Recuperado de http://www.scielo.cl/pdf/rchnut/v39n3/art05.pdf

Vio, F., Salinas, J., Montenegro, E., González, C. y Lera, L. (2014). Efecto de una intervención educativa en alimentación saludable en profesores y niños preescolares y escolares de la región de Valparaíso, Chile. Nutrición Hospitalaria, 29 (6), 1298-1304. Recuperado de http: / / scielo.isciii.es/pdf/nh/v29n6/10originalobesidad03.pdf

Vio, F., Lera, L., González, C., Yáñez, M., Fretes, G., Montenegro, E. y Salinas, J. (2015). Consumo, hábitos alimentarios y habilidades culinarias en alumnos de tercero a quinto año básico y sus padres. Rev Chil Nutr, 42 (4), 374-382. Recuperado de http: / / www.scielo.cl/scielo.php?pid $=$ S0717-75182015000400009\&script $=$ sci_

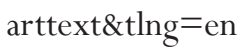

